\title{
Cavum trigeminale cephalocele associated with intracranial hypertension in an 18-month-old child: illustrative case
}

\author{
Giovanni Miccoli, MD, ${ }^{1,2}$ Domenico Cicala, MD, ${ }^{3}$ Pietro Spennato, MD, ${ }^{1}$ Alessia Imperato, MD, ${ }^{1}$ Claudio Ruggiero, MD, ${ }^{1}$ and \\ Giuseppe Cinalli, $M D^{1}$
}

\begin{abstract}
${ }^{1}$ Department of Neurosciences, Pediatric Neurosurgery Unit, Santobono-Pausilipon Children's Hospital, Naples, Italy; ${ }^{2}$ Department of Neurosciences, Reproductive and Odontostomatological Sciences, Division of Neurosurgery, University Federico II, Naples, Italy; and ${ }^{3}$ Department of Neurosciences, Division of Neuroradiology, SantobonoPausilipon Children's Hospital, Naples, Italy
\end{abstract}

\begin{abstract}
BACKGROUND Cavum trigeminale cephaloceles (CTCs) are characterized by a cystic appearance and extension of the posterolateral aspect of Meckel's cave into the superomedial portion of the petrous apex. A possible mechanism is a transient or sustained increase of intracranial pressure transmitted to Meckel's cave, leading to its secondary herniation, in the presence of predisposing local factors. The majority of CTCs are incidental findings. Among symptomatic cases, headache, trigeminal neuralgia, cerebrospinal fluid leak, vertigo, ataxia, facial numbness, hearing loss, diplopia, and other visual disturbances have been described.

OBSERVATIONS The authors describe a case of an 18-month-old male patient referred to their hospital with drowsiness, vomiting, left cranial nerve VI palsy, and papilledema. Neuroradiological investigation revealed the presence of a CTC with a swollen aspect of the left pontine hemisection and indirect signs of intracranial hypertension. The patient was managed with implantation of a ventriculoperitoneal shunt.

LESSONS CTCs are rare lesions that are difficult to recognize on neuroimaging, in which they can be mistaken for tumoral or inflammatory lesions. The most frequent asymptomatic forms should be considered as "leave-me-alone" lesions. In cases of intracranial hypertension, according to a supposed theory on the origin of CTCs, the authors recommend treating only the intracranial hypertension instead of performing more invasive surgical procedures, especially in pediatric patients.
\end{abstract}

https://thejns.org/doi/abs/10.3171/CASE21136

KEYWORDS cranial nerves; pseudotumor cerebri; ventriculoperitoneal shunt; middle fossa cephalocele

Cavum trigeminale cephaloceles (CTCS) are rare lesions that are characterized radiologically by their cystic appearance and extension of the posterolateral aspect of Meckel's cave into the superomedial portion of the petrous apex. ${ }^{1,2}$ Although termed "cephaloceles," these lesions usually do not contain brain tissue as is true for other cephaloceles of the skull base. A differential diagnosis should be done and should include cholesteatoma, trapped fluid, petrous apicitis, mucocele, cholesterol granuloma, and Meckel's cave schwannoma. ${ }^{3,4}$ Although usually incidental, associations with cerebrospinal fluid (CSF) leak, headache, trigeminal neuralgia, cranial nerve deficits, vertigo, and tinnitus have been reported. ${ }^{3}$
The exact etiology of CTCs remains unclear to date. The most widely accepted theory is that their origin is from increasing intracranial pressure (ICP) causing herniation of the meninges and CSF from the posterolateral region of the Meckel's cave into the petrous apex. ${ }^{1,5-7}$ CTCs can be acquired or congenital and either monolateral or bilateral. ${ }^{8,9}$ They are occasionally described together with the presence of an empty sella.,10,11 Herein we describe a case of an 18-month-old male patient referred to our clinic with left cranial nerve VI palsy, papilledema, and increased ICP who underwent ventriculoperitoneal shunt (VPS) placement. To date, to our knowledge, this is the youngest case diagnosed with a CTC.

ABBREVIATIONS CSF = cerebrospinal fluid; $\mathrm{CT}=$ computed tomography; $\mathrm{CTC}=$ cavum trigeminale cephalocele; $\mathrm{ICP}=$ intracranial pressure; $\mathrm{MRI}=$ magnetic resonance imaging; VPS = ventriculoperitoneal shunt.

INCLUDE WHEN CITING Published May 31, 2021; DOI: 10.3171/CASE21136.

SUBMITTED March 2, 2021. ACCEPTED March 18, 2021.

(C) 2021 The authors, CC BY-NC-ND 4.0 (http://creativecommons.org/licenses/by-nc-nd/4.0/). 

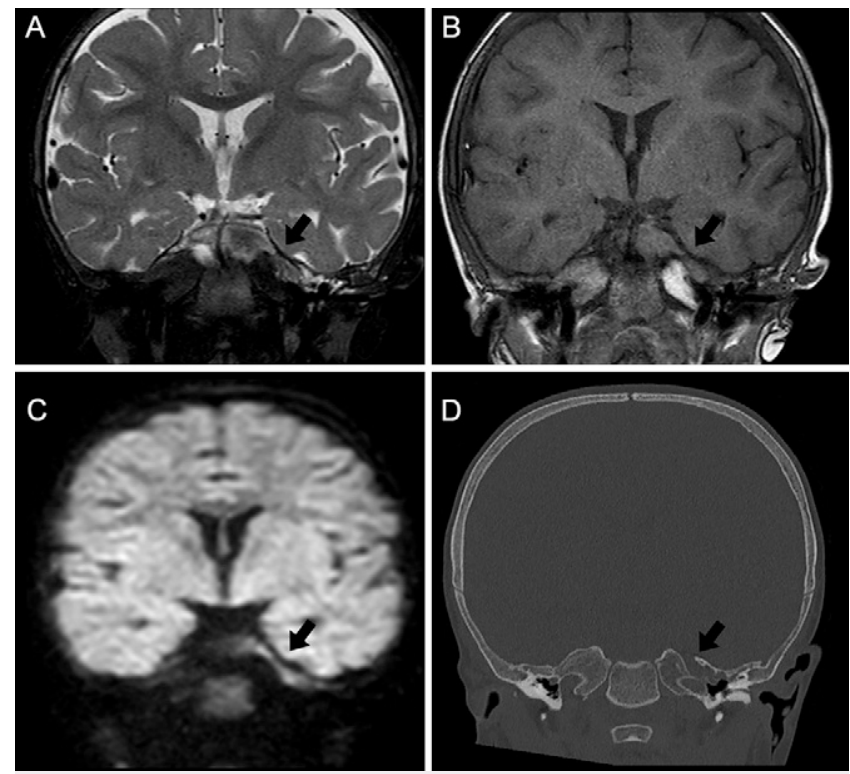

FIG. 1. Coronal fat-suppressed T2-weighted (A), T1-weighted (B), and diffusion-weighted imaging (DWI) (C) MRI scans and a coronal CT image (D). The images show an inhomogeneous and atypical appearance of the superolateral profile of the petrous apex, such as a recess containing the Gasser ganglion in its medial portion. The arrows indicate the trigeminal root traction at the ostium of the recess, with signs of acute suffering on the DWI study. The coronal CT shows a corresponding bone remodeling cavity with direct communication with the intracranial compartment, suggesting the hypothesis of a CTC with ectopic Meckel cave and trigeminal incarceration.

\section{Illustrative Case}

An 18-month-old male presented with a 1-day history of irritability, failure to thrive, drowsiness, vomiting, and left cranial nerve VI palsy. Three months before admission, he experienced otitis media, which had been treated with antibiotics. Clinical examination revealed left cranial nerve VI palsy, bilateral papilledema, and opacification of the left tympanic membrane. Computed tomography (CT) revealed a swollen aspect of the left pontine hemisection and secondary restriction of the homolateral pre- and peripontine cistern. Bone erosion of the left petrous apex together with slight enlargement of the ventricular system was evident. Magnetic resonance imaging (MRI) (Figs. 1 and 2A) confirmed an area with inhomogeneous content along the superolateral profile of the left petrous apex, such as an excavated Meckel's cave, with tissue bands mixed with small fluid areoles and peripheral contrast enhancement, probably secondary to congestive phenomena. Highresolution sequences clearly defined the distortion of the pons caused by trigeminal root traction at this bony recess. Furthermore, an asymmetrical bone structure of the petrous apex and of the sphenoid was detected (Fig. 3), as well as the representation of Meckel's cave and the cavernous sinuses. These findings suggested a diagnosis of sectorial bone hypoplasia and CTC, with an ectopic Meckel's cave and trigeminal encasement. MRI also revealed an empty sella and bilateral ectasia of the optic nerve sheaths caused by intracranial hypertension, as well as a bilateral gap of the transverse sinuses (Fig. 4A).

The patient underwent lumbar puncture, which revealed an opening pressure of $45 \mathrm{~mm} \mathrm{Hg}$, with transitory improvement of symptoms. A left tympanic stent was also placed by an ear, nose, and throat surgeon to drain the left middle ear. A new brain MRI scan (Fig. 2B) documented a mild reduction of optic sheaths. However, the patient's symptoms recurred, and he underwent a second lumbar puncture, which also showed intracranial hypertension $(70 \mathrm{~mm} \mathrm{Hg}$ ). Finally, a VPS was placed because of persisting cranial nerve VI palsy and drowsiness (left frontal access, small programmable valve). Postoperatively, the patient improved dramatically with rapid resolution of the cranial nerve palsy $\mathrm{VI}$ and slow regression of the papilledema.

Postoperative brain MRI documented the progressive reexpansion of the prepontine cistern and the restoration of CSF content in Meckel's cave. The patient was then discharged. Two weeks later, symptoms of intracranial hypertension recurred. A CT scan showed disconnection of the ventricular catheter from the valve. The patient underwent surgical revision of the shunt, with resolution of symptoms.

One-month follow-up postoperative MRI revealed very mild enlargement of the Meckel's cave cephalocele with slight reduction of trigeminal and correspondent root entry zone attraction at the left petrous apex caused by gliomalacic phenomena and good recanalization and visibility of both transverse sinuses (Fig. 4B).

\section{Discussion}

\section{Observations}

The petrous apex has a close relationship with cranial nerves, the auditory apparatus, the carotid artery, the cavernous sinus, and the other skull base venous plexuses. Several lesions can be found at this precise anatomical location: cholesteatoma, cholesterol granuloma, primary and secondary tumors such as rhabdomyosarcoma, Ewing sarcoma, metastatic neuroblastoma, and other infectious or inflammatory lesions. ${ }^{4} \mathrm{~A}$ differential diagnosis based on imaging findings is essential because the anatomical location is difficult to approach even for a biopsy. $3,4,12$

CTCs are very rare lesions thought to arise from a posterolateral dural or arachnoid protrusion into the petrous apex. ${ }^{1,13}$ The etiopathogenetic mechanism at the origin is not clear at all. A possible mechanism is a transient or sustained increase of ICP transmitted to Meckel's cave, leading to its secondary herniation. ${ }^{1,13}$ Anyway, CTC is difficult to explain by altered mechanisms of CSF pressure and dynamics alone without assuming the presence of local factors at the petrous apex. A thin bony plate covered by a thin dural layer could be the site of dehiscence following intracranial hypertension, also because of the spongy bone configuration, sometimes pneumatized. ${ }^{1,6}$ Schlosser and Bolger explained skull base erosions as an attempt to alleviate increased ICP in highly pneumatized skull bases. Moreover, our findings of elevated pressure at lumbar puncture are equally consistent. ${ }^{7}$ Stark et al. believe there is CSF passage into the cisternal mesenchyme during gestational life, resulting in arachnoid membrane splitting, leading to the formation of a CSFcontaining cyst. ${ }^{5}$

The majority of CTCs are incidental findings usually considered as "leave-me-alone" lesions. Among symptomatic cases, headache, trigeminal neuralgia, CSF leak, vertigo, ataxia, facial numbness, hearing loss, diplopia, and other visual disturbances have been described. ${ }^{3,6,9,10,13}$

Our patient was referred to us with irritability, failure to thrive, drowsiness, vomiting, and left cranial nerve VI palsy. To our knowledge, he represents the youngest patient diagnosed with CTC to date. Previously, only one case of recurrent meningitis had been reported in a 2-year-old patient. ${ }^{14}$ 

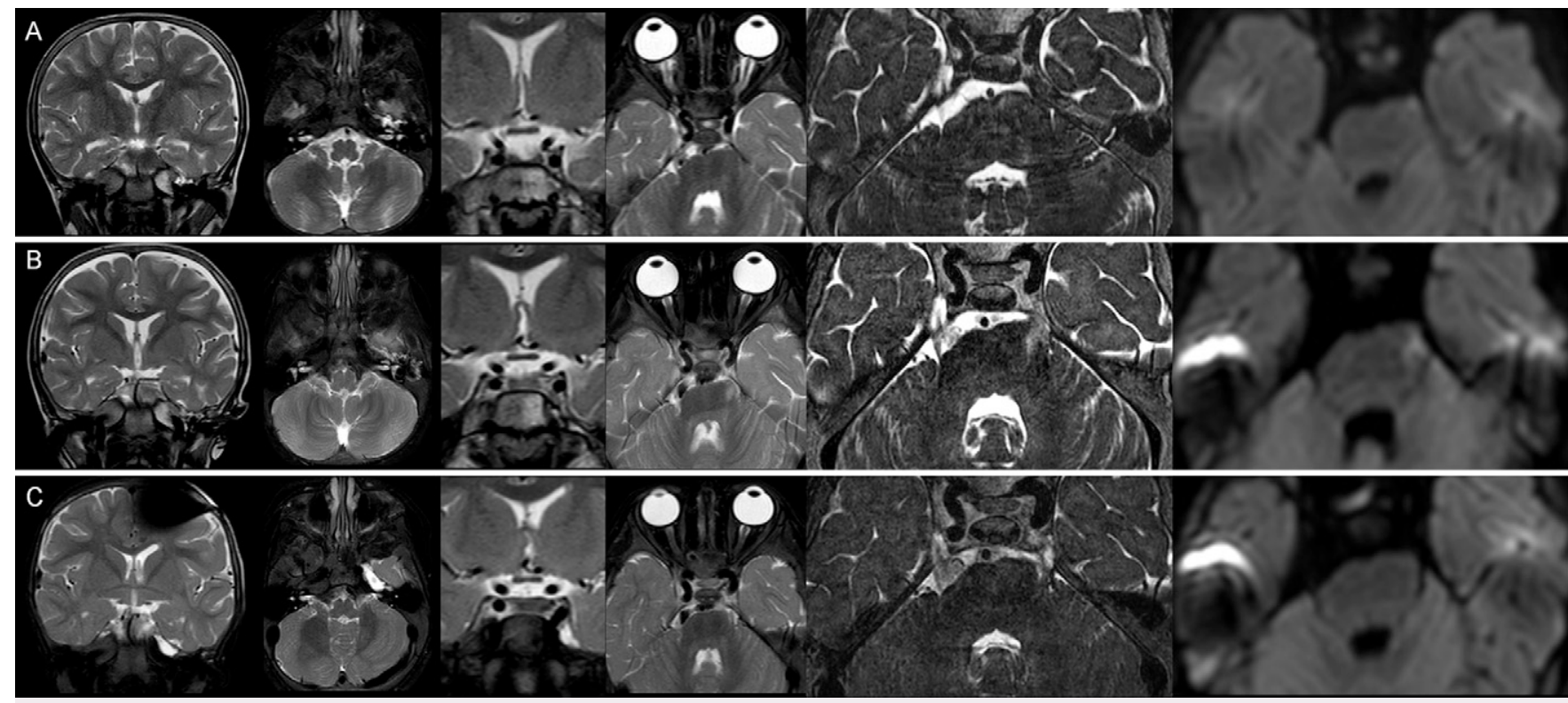

FIG. 2. Coronal and axial T2-weighted MRI scans centered on the petrous apex (left); coronal and axial T2-weighted MRI scans at the juxtasellar level (center); axial Driven Equilibrium high-resolution and DWI MRI scans (right). Evolution of neuroradiological findings at the onset (A) and at follow-up after 1 week (B) and 3 months (C). A: Images show CTC in the left petrous apex with retraction of the trigeminal root and distortion of the pons; note the correlated expansion of the subarachnoid space of the ipsilateral hemispheric convexity; note also the dilatation of the perioptic subarachnoid space and the empty sella, suggestive of intracranial hypertension. B: Early MRI evaluation after lumbar puncture shows partial regression of intracranial hypertension. It also shows progressive acute tissue damage in the left trigeminal root entry zone (REZ), with restricted diffusion on a DWI study. C: Late follow-up study after VPS placement documents regression of retraction phenomena and progressive reexpansion of the prepontine cistern. It shows restoration of CSF content in the ectopic Meckel's cave as a fluid-filled CTC. Note the progressive gliomalacic involution of the trigeminal REZ along the pontine anterolateral profile.

A combination of CT and MRI scans could be effective to differentiate CTCs from other petrous apex lesions. A CT scan allows detection of invasive erosions of petrous bone structures. In our case, bony remodeling at the left petrous apex was clearly evident.

On brain MRI, CTCs display a CSF-like signal in all sequences with hypointensity on T1-weighted images, hyperintensity on T2weighted images, and no restriction for diffusion-weighted imaging scans. Contrast enhancement is not typical, even if peripheral enhancement has been described. ${ }^{1,2,11,13}$ Our patient showed a unilateral CTC; the pathogenesis of his CTC seemed to be explained by petrous apex sectorial hypoplasia.

The coexistence of a CTC and an empty sella has been addressed. Previous publications reported on how impaired CSF dynamics and increased ICP could lead to herniation of meninges and CSF into the sella turcica and the petrous apex. ${ }^{3,10,11}$ In our patient, venous disease may have played a major role in inducing intracranial hypertension, as suggested by the absence of signal in the venous sinuses on MRI. However, we think that venous obstruction may be, at least in part, secondary to distortion of the venous anatomy secondary to intracranial hypertension: Venous obstruction further increases venous pressure, CSF pressure, and ICP in a vicious cycle. Shunting, decreasing ICP, reverses this vicious cycle, allowing reopening of the sinuses.

Management of these lesions is not standardized. No treatment is recommended for incidental and totally asymptomatic findings. ${ }^{8,13}$

When surgical intervention is needed, what is recommended to be done? Open microsurgical fenestration or marsupialization have been described. ${ }^{3,12}$ The main surgical approach adopted for this purpose (i.e., infracochlear, retrosigmoid, retrolabyrinthine, middle fossa) is even more invasive and not at all free from complications.

\section{Lessons}

According to the supposed mechanism of the origin of CTCs (in the presence, or not, of congenital or acquired petrous apex bone alterations), we recommend treating only the increased ICP. In our case, the decision was relatively easy. The patient's clinical history (previous middle ear infection), symptoms (headache, irritability), clinical findings (cranial nerve VI palsy, papilledema), radiological findings (dilated optic nerve sheaths, empty sella, steno-occlusion of both transverse sinuses), and ancillary tests (high CSF pressure at lumbar puncture with manometry) were all in favor of a diagnosis of associated idiopathic intracranial hypertension (pseudotumor cerebri), which has already been described in association with cephaloceles of the petrous apex. ${ }^{3,8,11} \mathrm{~A}$ decision was made to address uniquely the predominant clinical condition (idiopathic intracranial hypertension) with a standard treatment (navigated frontal VPS), disregarding any hypothesis of surgical treatment of the cephalocele of the petrous apex.

An important radiological sign that we observed on MRI following the first lumbar puncture was, even if mild in size, the reduction of optic nerve sheath ectasia. After the patient underwent VPS placement, we observed successful improvement of his clinical and neurological condition together with initial recovery of the cranial nerve VI palsy. At his last follow-up, the patient was still doing well and had totally recovered from his cranial nerve deficit. Brain MRI, showing 

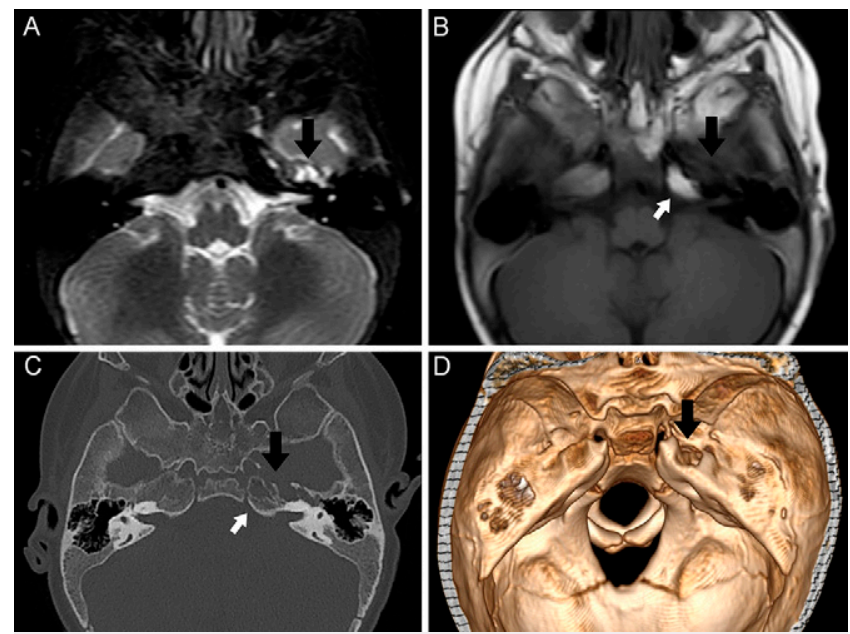

FIG. 3. Axial fat-suppressed T2-weighted (A) and T1-weighted (B) MRI scans and axial (C) and volume rendering (VR) (D) CT images. MRI studies show area with inhomogeneous content in the lateral slope of the left petrous apex, with tissue mixed with small fluid areoles (black arrows, $\mathbf{A}$ and $\mathbf{B}$ ). Note the signal asymmetry of the spongy bone in the petrous apex and sphenoid, with a predominant adipose component of the left hemisections, showing T1-weighted hyperintensity (white arrow, B). The CT studies highlight the lithic area with demarcated margins at the petrous apex, suggestive of CTC (black arrows, $C$ and D). The VR image shows the excavating lesion remodeling the bone of the petrous apex. Axial CT image also shows an atypical structure of the spongy bone at the same level (white arrow, C), which supports the hypothesis of a sectorial hypoplasia or dysplasia of the skull base.

disappearance of the empty sella and bilateral recanalization of the transverse sinuses, confirmed the resolution of increased ICP.

In conclusion, CTCs are rare lesions that are difficult to recognize on neuroimaging. The most frequent asymptomatic forms should be considered as "leave-me-alone" lesions. According to the supposed theory of the origin of this clinical condition, we recommend treating
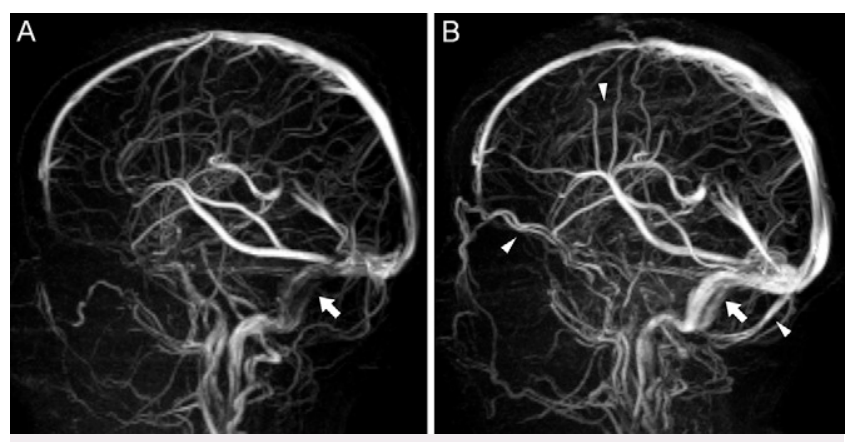

FIG. 4. Pre- and postoperative magnetic resonance (MR) venography (three-dimensional phase contrast). Initial MR venography (A) shows bilateral transverse sigmoid sinus gap related to intracranial hypertension (arrow). The last MR venography study (B) highlights a complete restoration of flow signal of both lateral sinuses (arrow); it also reveals an increased representation of other veins (arrowheads), such as the occipital sinus, the superior ophthalmic veins draining into the petrous sinus, and some cortical veins draining into the Labbé veins. These findings are suggestive of improvement of venous circulation resulting from resolution of hypertension. only the eventually associated increased ICP instead of performing more invasive surgical procedures, especially in pediatric patients.

\section{References}

1. Alorainy IA. Petrous apex cephalocele and empty sella: is there any relation? Eur J Radiol. 2007;62(3):378-384.

2. Chapman PR, Shah R, Curé JK, Bag AK. Petrous apex lesions: pictorial review. AJR Am J Roentgenol. 2011;196(suppl 3):WS26-WS37, S40-S43.

3. Isaacson B, Coker NJ, Vrabec JT, et al. Invasive cerebrospinal fluid cysts and cephaloceles of the petrous apex. Otol Neurotol. 2006;27(8):1131-1141.

4. Radhakrishnan R, Son HJ, Koch BL. Petrous apex lesions in the pediatric population. Pediatr Radiol. 2014;44(3):325-339.

5. Stark TA, McKinney AM, Palmer CS, et al. Dilation of the subarachnoid spaces surrounding the cranial nerves with petrous apex cephaloceles in Usher syndrome. AJNR Am J Neuroradiol. 2009;30(2):434-436.

6. Warade AG, Misra BK. Petrous apex cephalocele presenting with cerebrospinal fluid rhinorrhea in an adult. $\mathrm{J}$ Clin Neurosci. 2016;25:155-157.

7. Schlosser RJ, Bolger WE. Significance of empty sella in cerebrospinal fluid leaks. Otolaryngol Head Neck Surg. 2003;128(1):32-38.

8. Alkhaibary A, Musawnaq F, Almuntashri M, Alarifi A. Bilateral petrous apex cephaloceles: is surgical intervention indicated? Int $\mathrm{J}$ Surg Case Rep. 2020;72:373-376.

9. Boppel T, Bendszus M, Bartsch AJ. Excavating Meckel's cave: cavumtrigeminale-cephaloceles (CTCs). J Neuroradiol. 2015;42(3):156-161.

10. Jamjoom DZ, Alorainy IA. The association between petrous apex cephalocele and empty sella. Surg Radiol Anat. 2015;37(10): $1179-1182$.

11. Hatipoğlu HG, Cetin MA, Gürses MA, et al. Petrous apex cephalocele and empty sella/arachnoid cyst coexistence: a clue for cerebrospinal fluid pressure imbalance? Diagn Interv Radiol. 2010;16(1):7-9.

12. Isaacson B, Kutz JW, Roland PS. Lesions of the petrous apex: diagnosis and management. Otolaryngol Clin North Am. 2007;40(3): 479-519.

13. Moore KR, Fischbein NJ, Harnsberger HR, et al. Petrous apex cephaloceles. AJNR Am J Neuroradiol. 2001;22(10):1867-1871.

14. Motojima T, Fujii K, Ishiwada N, et al. Recurrent meningitis associated with a petrous apex cephalocele. J Child Neurol. 2005;20(2): 168-170.

\section{Disclosures}

The authors report no conflict of interest concerning the materials or methods used in this study or the findings specified in this paper.

\section{Author Contributions}

Conception and design: Spennato, Miccoli, Cicala, Ruggiero, Cinalli. Acquisition of data: Miccoli, Cicala, Imperato, Ruggiero. Analysis and interpretation of data: Cicala, Ruggiero, Cinalli. Drafting the article: Miccoli, Cicala, Imperato. Critically revising the article: Spennato, Cicala, Ruggiero, Cinalli. Reviewed submitted version of manuscript: Spennato. Approved the final version of the manuscript on behalf of all authors: Spennato. Administrative/technical/material support: Cicala, Imperato. Study supervision: Ruggiero.

\section{Correspondence}

Pietro Spennato: Santobono-Pausilipon Children's Hospital, Naples, Italy. pierospen@gmail.com. 